\title{
ULTRASONIC SPECTROSCOPY OF THE
}

\section{ACCEPTOR GROUND STATE IN CUBIC SEMICONDUCTORS}

\author{
Kurt Lassmann and Heinrich Zeile \\ Physikalisches Institut, Universität Stuttgart \\ Pfaffenwaldring 57, D-7000 Stuttgart 80, FRG
}

\section{INTRODUCTION}

Phonons are effectively scattered by electrons and holes bound to donors and acceptors in semiconductors at low temperatures corresponding to the relatively large deformation potential constants involved. This has been found over twenty years ago in measurements of the thermal resistivity and the ultrasonic attenuation of bound donor states in Germanium. Since then many phonon scattering experiments on both donors and acceptors have been carried out and a lot of results have been obtained. Much of the progress in understanding is due to the progress of industry and research in manufacturing pure and well-defined crystals. This applies particularly to the acceptor ground state which is very sensitive to crystal imperfection. In the following we will discuss ultrasonic measurements on p-type semiconductors reflecting spontaneous and defect sensitive modifications of the acceptor ground state.

For the bound states of shallow impurities in semiconductors a hydrogenic model is justified where the wavefunctions are constructed from the neighbouring band states leading to relatively small ionization energies $E_{A} \simeq 10$ to $100 \mathrm{meV}$ and relatively large Bohr radii $r_{B^{\alpha 1}} / \sqrt{ } E_{A} \sim 100$ to $10 R$. This means that these states are extended point defects. The ground state of acceptors in cubic semiconductors is a fourfold degenerate $\Gamma_{8}$ state that may be split by elastic or electric fields into two Kramers doublets. A magnetic field will lift the degeneracy completely. Such a $\Gamma_{8}$ state should, in principle, show an instability against spontaneous deformation, viz. a JahnTeller effect. However, because of the extended wavefunction, a Jahn-Teller effect is expected to be weak for shallow acceptors, it 
should be more pronounced for deeper acceptors with their smaller Bohr-radii.

In fact, for two acceptors, namely GaAs(Mn) $\left(E_{A}=110 \mathrm{meV}\right.$, $\left.r_{B}=10.4 \AA\right)$ and $\mathrm{Si}(\mathrm{In})\left(E_{A}=156 \mathrm{meV}, r_{B}=7.3 \AA\right)$ we have found resonance energies at $3.1 \mathrm{meV}$ and $4.2 \mathrm{meV}$ respectively, in reasonable accordance with first calculations of the problem ${ }^{2}$. Experimentally, these values have been obtained from an analysis of the ultrasonic relaxation attenuation ${ }^{3} 4$, from thermal conductivity measurements ${ }^{5}$, and, in the case of $\mathrm{Si}(\mathrm{In})$, also from experiments with quasimonochromatic phonons ${ }^{6}$ and from a satellite in luminescence ${ }^{7}$.

The vibronic ground state of the system will exhibit full symmetry, that is, it will also be a $\Gamma_{8}$ state. Internal elastic and electric fields originating from crystal defects will lead to a distribution of splittings which, in turn, may be regarded as an indicator of residual defects. Such a distribution of two-level systems is quite analogous to that encountered in glasses, and we are faced with the same problems as to the form of the distribution, the relaxation times and the interaction between defects.

\section{THE DISTRIBUTION OF SPLITTINGS}

The form of the distribution function may be obtained by measuring the resonant attenuation in a wide frequency range: Since the attenuation $\alpha$ is proportional to the spectral density $N(\delta)$ of the splittings $\delta$ at a certain frequency $v=\delta / h$, the distribution is thus probed in the corresponding frequency range.

For the resonant absorption we have

$$
\begin{aligned}
& \alpha(v, T)=\int N(\delta) \alpha(\delta, v, T) d \delta \\
& \alpha(\nu, T) \propto \int\left[\tanh (\delta / 2 k T) D_{\text {eff }}^{2}(\delta) N(\delta) v \Gamma(\delta, T) /\left[(h v-\delta)^{2}+\Gamma^{2}(\delta, T)\right]\right] d \delta
\end{aligned}
$$

If the linewidth $\Gamma(\delta, T)$ of the resonance line is small enough compared to the variation of $\mathrm{N}(\delta), \mathrm{D}_{\text {eff }}(\delta)$ we can write:

$$
\alpha(\nu, T) \propto\left[N(\delta=h v) D_{\text {eff. }}^{2}(\delta=h v)\right]\left(v^{2} / T\right)
$$

for $h v<4 T$. The effective coupling constant $D_{\text {eff }}$ is a combination of the two well-known deformation potential constants of the ground state corresponding to the "average" symmetry of the disturbing field. Assuming that this "average" symmetry does not vary with $\delta$ we may write $\alpha(\nu) / \nu^{2} \propto N(\delta=h \nu)$ at a fixed temperature. In Fig.1 we have plotted the variation of $\alpha(\nu) / \nu^{2}$ in the frequency range $0.5 \mathrm{GHz} \leq U \leq 4.2 \mathrm{GHz}$ for a boron doped high quality silicon crystal. Under the assumption that $D_{\text {eff }}$ is constant and that $\alpha(v) / v^{2}$ is negligibly small outside our measuring range we can integrate the 


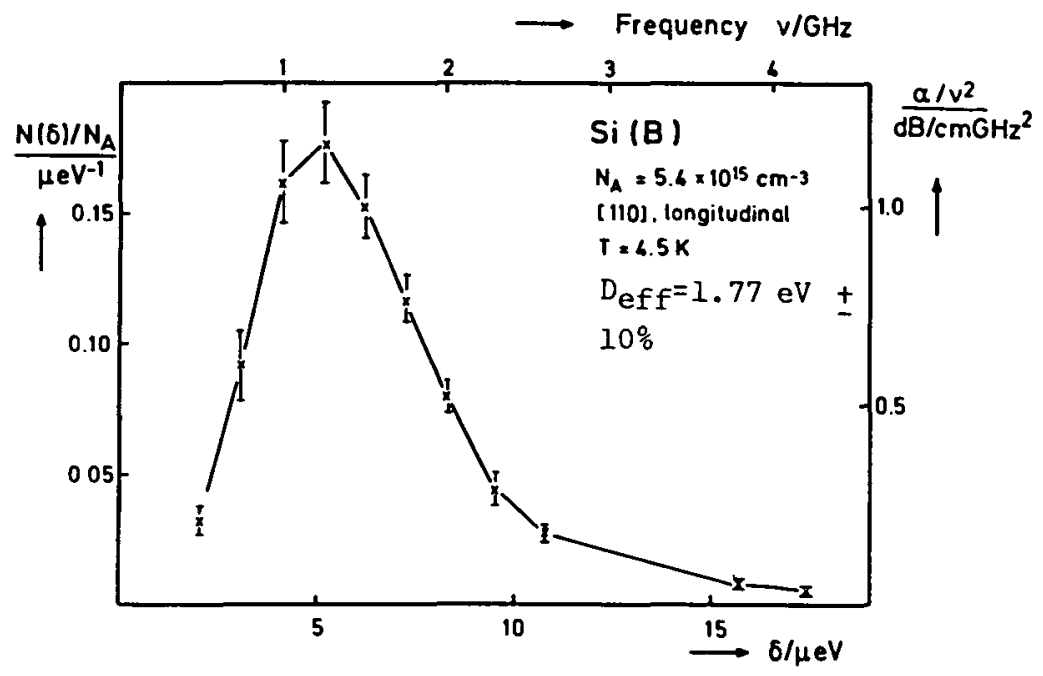

Fig. 1: Distribution of splittings of the boron acceptor ground state for a high quality silicon crystal.

area to obtain $\mathrm{N}_{\mathrm{A}} \cdot \mathrm{D}^{2}$ eff where $\mathrm{N}_{\mathrm{A}}$ is the known acceptor concentration. The value $D_{\text {eff }}=1.8$ eff seems reasonable in comparison with the two deformation potential constants $D_{u}^{\mathrm{a}}=2.4 \mathrm{eV}$ and $\mathrm{D}_{\mathbf{u}}^{\mathrm{a}}=3.9 \mathrm{eV}$. In applying uniaxial pressure we have verified for a similar crystal that there is no peak in the distribution function at $\delta=0$. Measuring the attenuation for other directions and polarizations one may separate the different elastic constants involved to get additional information on $\mathrm{D}_{\mathrm{eff}}$. In Fig. 2 the distribution functions are shown for a series of high quality silicon crystals of varying boron concentrations. They are all similar with maxima between $1 \mathrm{GHz}$ and 2 $\mathrm{GHz}$, falling rapidly of $f$ at low frequencies. With growing boron concentration there is a tendency to flatten the maximum and to broaden the distribution to higher energies. All these crystals (supplier Wacker Chemitronic, Burghausen, F.R.G.) are floating zone-pulled, dislocation-free, with low carbon and oxygen contents (below $1015 \mathrm{~cm}^{-3}$ to $1016 \mathrm{~cm}^{-3}$ typically). The pulling velocity was high enough to avoid detectable clusters of selfinterstitials ("swirl-free" crystals), however, the concentration of selfdefects is unknown. It may be as high as $10^{17} \mathrm{~cm}^{-3}$. ( The concentration of selfdefects may be reduced in pulling the crystal at extremely low velocity, where the defects have time to diffuse out of the crystal during growth ${ }^{8}$ ). The crystal of Fig. 1 differed from those of Fig. 2 in that the pulling conditions were such as to produce an even higher homogeneity of the crystal.

In less pure crystals (Czochralski-grown $S i(B)$ and $S i(I n)$ with 


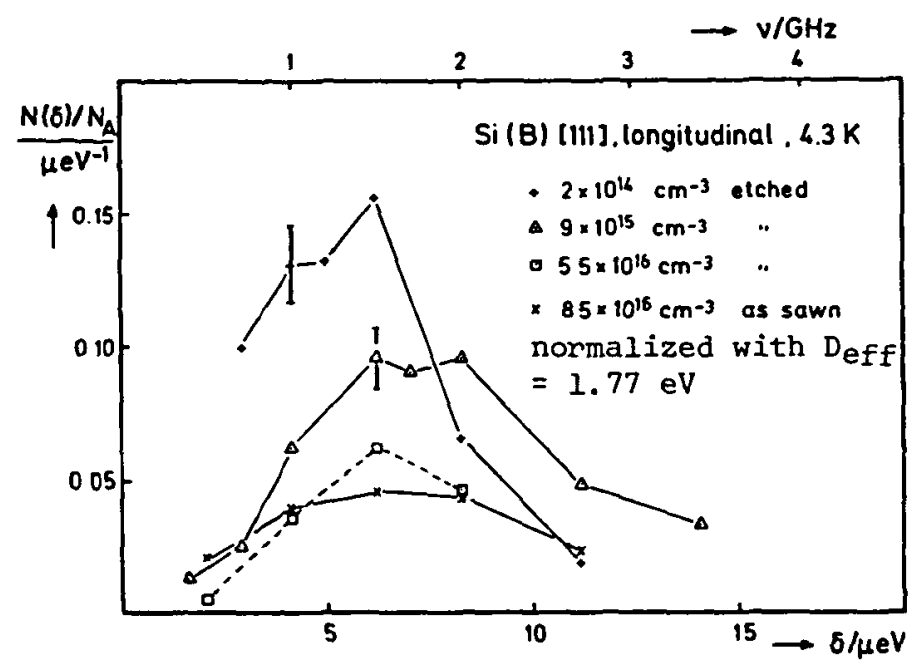

Fig. 2: Distribution of splittings for various boron concentrations.

high carbon and oxygen content) we find only a rise in $\alpha / \nu^{2}$ indicating a maximu beygnd our limit of about $4 \mathrm{GHz}$. Earlier we have found for $\mathrm{GaAs}\left(\sim 10^{18} \mathrm{~cm}^{-3} \mathrm{Mn}\right)^{3} \alpha / \nu^{2}$ to be practically constant from 0.4 to $2.0 \mathrm{GHz}$ and also for Ge of various doping ${ }^{9}$ between $8 \times 10^{15} \mathrm{~cm}^{-3}$ and $3 \times 10^{16} \mathrm{~cm}^{-3} \alpha / \mathrm{v}^{2}$ constant between $1.2 \mathrm{GHz}$ and $2.5 \mathrm{GHz}$ indicating a width of the distribution of about $50 \mathrm{\mu eV}$ and $100 \mathrm{\mu eV}$ respectively. (In contrast, for a very pure Ge(2xI014 $\mathrm{cm}-3 \mathrm{Ga})$ crystal

grown and kindly supplied by E.E. Haller of Lawrence Berkeley Laboratory, California, we find a distribution even narrower than that of Fig. 1 with maximum near $1 \mathrm{GHz}$.)

So far, we don't know the reason for the residual linewidth of the purest crystals. We shall shortly discuss three possibilities:

(i) Though there is some correlation with boron concentration visible in our results, ground state splitting due to wave function overlap seems improbable, since we would expect a monotonous exponential dependence of the splitting on mean acceptor distance for this range of small energies ${ }^{10}$. (ii) Electric fields from residual compensating donors should be too small for the low concentrations of residual donors $\left(\sim 10^{12} \mathrm{~cm}^{-3}\right.$ in the purest crystal). In Ref. 11 we overestimated the electric field in our calculation. Furthermore, at low temperatures the ionized donors and acceptors should not be uncorrelated, as assumed ${ }^{12}$ in that calculation, because a nearest neighbour correlation will be energetically more favourable. Such a correlation will lead to a further reduction of the electric field, as we have found by a computer simulation. (iii) Strain fields from point defects would be expected at relatively high concentrations. In Fig. 3 


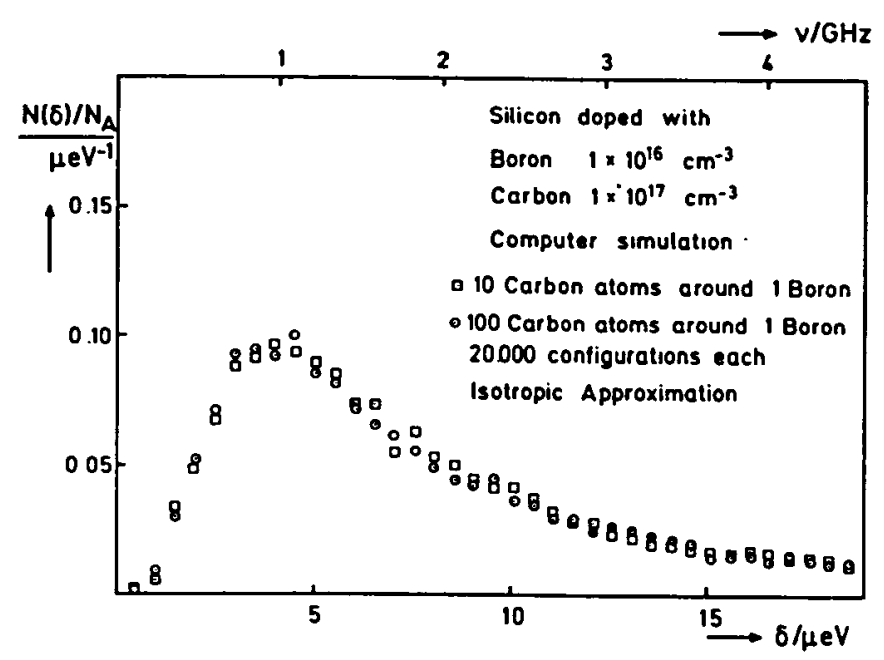

Fig. 3: Computer simulation of splitting distribution due to substitutional carbon.

we have plotted the distribution of splitting resulting from a computer simulation for substitutional carbon with an elastic strength $\mathrm{A}=-0.85 \AA^{3}$ corresponding to its covalent radius in an isotropic approximation for the crystal. Since the elastic strength of boron is much smaller, we see that neither the boron nor the carbon or oxygen content should be responsible for the residual splitting. Since the concentration of intrinsic defects or their agglomeration is unknown, at the present state of our investigation we cannot say whether selfdefects or some other defects or perhaps some effects intrinsic to the isolated acceptor are responsible for the residual splitting.

THE CRITICAL INTENSITY

To separate the resonant attenuation $\alpha_{\text {res }}$ from relaxation attenuation and geometrical effects we make use of the possibility of saturation at high acoustic intensities. From these intensity dependent measurements we obtain the critical intensity $\mathrm{J}_{\mathrm{c}}$.

In a Bloch equation formalism we obtain from $J_{c}$ the product $\tau_{1} \cdot \tau_{2}$ of the "spin"-lattice or energy relaxation time $\tau_{1}$ and the transverse or dephasing relaxation time $\tau_{2}$

$$
J_{c} \propto 1 /\left(\tau_{1} \cdot \tau_{2}\right)
$$

If only "spin"-lattice relaxation were effective, one might assume 
$\tau_{1} \cdot \tau_{2}=2 \tau^{2}{ }^{13}$; i.e. $I_{c}$ should not depend on $N_{A} \cdot A$ dynamical interaction between the acceptors should shorten $\tau_{2}$, leading to a larger $I_{c}$ for smaller mean distances between the acceptors. In Fig. 4 we have plotted the critical intensity for various acceptor concentrations. We see that below about $10^{16} \mathrm{~cm}^{-3}$ the critical intensity does not depend on $N_{A}$. From $I_{c}=10^{-4} \mathrm{~W} / \mathrm{cm}^{2}$ we estimate $\tau_{1} \cdot \tau_{2} / \sqrt{2}=\tau_{1}=$ $1.4 \times 10^{-8} \mathrm{sec}$, whilst from our measurement of the relaxation attenua-

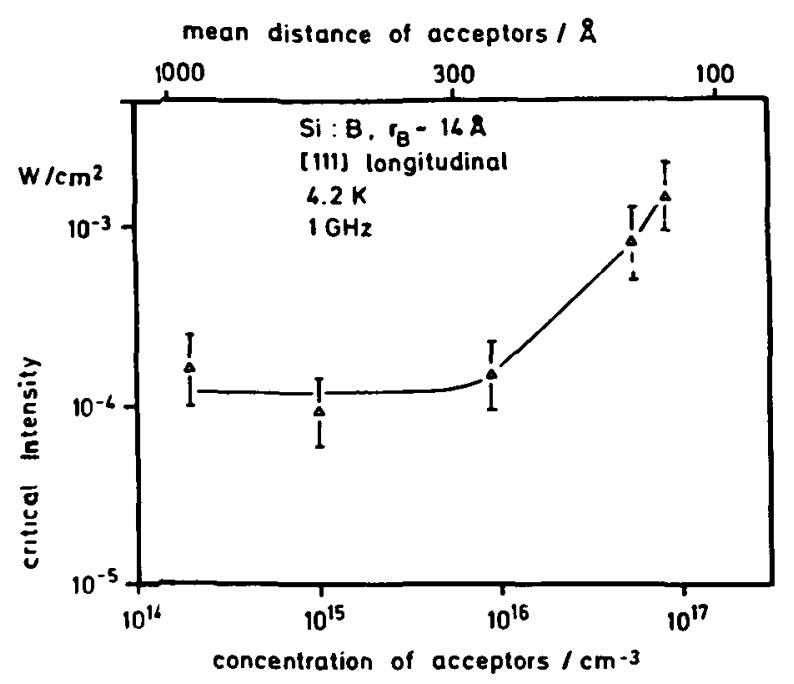

Fig. 4: Critical intensity as a function of average acceptor distance.

tion we have $\tau_{1}=1.3 \times 10^{-8} \mathrm{sec}$, which means, that for average distances larger than about $300 \AA$ the critical intensity is determined by "spin"-lattice relaxation alone. For smaller distances $d$ the critical intensity rises roughly as $1 / \mathrm{d}^{3}$, as one might expect for a dipolar interaction ${ }^{13} 14$. However, applying the formula for spectral diffusion by elastic dipolar interaction given in 14 for our experimental conditions (Raman/Orbach relaxation, narrow distribution), we arrive at $\Delta \omega / \mathrm{Hz}=5.6 \cdot 10^{-8}\left(\mathrm{~N}_{\mathrm{A}} / \mathrm{cm}^{-3}\right)$ (independent on temperature) which leads to $\tau_{2}$ being too small by an order of magnitude. Also the temperature dependence of $J_{c}$ varies more slowly than expected. We are, therefore, preparing experiments for a direct determination of $\tau_{2}$.

Applying uniaxial pressure will "shift" the distribution and reduce the concentration of acceptors on speaking terms. In our first experiments we have seen no effect on $I_{c}$ at $4.2 \mathrm{~K}$ reducing 
$\alpha(\nu) / \nu^{2}$ by about a factor of five. This is as expected since the energy-independent Raman/Orbach relaxation is dominant in this range so that spectral diffusion should remain unchanged and on the other hand because the acceptors on speaking terms are at any rate relatively minor in number to have an influence on spectral diffusion.

For the narrow distribution in a magnetic field acoustic paramagnetic resonance can be seen and lines be resolved at $4 \mathrm{GHz}$. Also EPR at $10 \mathrm{GHz}$ is possible in these clean crystals, as was recently shown $^{15}$. By detailed investigation of the APR at somewhat higher frequencies we should get additional information on the symmetry and distribution of the random splittings of the acceptor ground state.

We hope that investigations of this kind may help to some practical informations concerning residual crystal defects as well as to a better understanding of the dynamical processes connected with the two-level systems.

This work has been supported by the Deutsche Forschungsgemeinschaft (SFB 67), Stuttgart.

\section{REFERENCES}

1. For reference to earlier work see

H. Tokumoto and T. Ishiguro, Phys. Rev. B 15:2099 (1977)

2. E. Sigmund and K. Lassmann, These Proceedings

3. K. Lassmann and Hp. Schad, Solid State Commun. 19:599 (1976)

4. Hp. Schad and K. Lassmann, Phys. Lett. 56A:409 (1976)

5. A. de Combarieu and K. Lassmann, Phonon Scattering in Solids, Plenum Press, N.Y. (1976)

6. H. Schenk, W. Forkel and W. Eisenmenger, Verhandl. DPG 1/1978; page 328

7. R. Sauer, W. Schmid and J. Weber, Solid State Commun. 27:705 (1978)

8. P.J. Roksnoer, W.J. Bartels and C.W.T. Bulle, J. Cryst. Growth $35: 245$ (1976)

9. E. Ortlieb, Hp. Schad and K. Lassmann, Solid State Commun. 19:599 (1976)

10. E. Kaczmarek and Z.W. Gortel, Phys. Rev. B10:2535 (1974)

11. H. Zeile, O. Mathuni and K. Lassmann, J. de Physique L40:53 (1979) ( $I_{c}$ of Fig.2 has to be increased for the overlap of the ultrasonic pulse train by a factor of 2.)

12. D. Larsen, Phys. Rev. B13:1681 (1976)

13. J. Joffrin and A. Levelut, J. Physique 36:811 (1975)

14. J.L. Black and B.J. Halperin, Phys. Rev. B16:2879 (1977)

15. H. Neubrand, phys. stat. sol. (b) 86:269 (1978) 


\section{DISCUSSION}

R. Collella: These impurities are on the average inhomogeneously distributed in these crystals. I was wondering whether you have considered using crystals like silicon in which the doping is achieved by neutron irradiation, and you can have the same kind of doping with the same percentages, but you are sure that it is much more homogeneous. It would be interesting to compare if you get the same results.

K. Lassmann: By this method you can obtain donors by transportation of silicon into phosphorous, and this we used already in one case. For acceptors I don't know of a possibility to make it more homogeneous. What we tried to do was to look with an infrared microscope and in the case of some silicon-indium crystals where we also have some broader distribution there we saw some striations. With these silicon crystals, especially the first one I showed, it would be very inhomogeneous, the suppliers told me but they didn't tell me how they told it. There we didn't see with the microscope any striations, but we cannot tell any more about that. What we want to do is to see if there is any inhomogeneity correlated say with the pulling direction so we might work in different directions if we are allowed to do it. For instance, under pressure we could see whether there are three types of strain. This is really a problem. Also association between defects might be a problem.

J. P. Harrison: Could you measure the strain dipole moment by applying a stress and then repeating your measurements?

K. Lassmann: It is potentially given by the deformation potential constants which have been measured. 